\title{
ANESTESIA EN TERAPIA ELECTROCONVULSIVA (TEC)
}

\author{
Fernando Reyes R. ${ }^{1}$, Hernán Arancibia I. ${ }^{2,}$ Alejandro González A. ${ }^{3}$, \\ Carolina Pérez O. ${ }^{4}$ y Francisca Elgueta Le-Beuffe ${ }^{5}$
}

Key words: Electroconvulsive therapy, Anesthesiology, Psychiatry.

\section{INTRODUCCIÓN}

La terapia electroconvulsiva (TEC) es un procedimiento seguro usado por la psiquiatría para tratar ciertas patologías graves y resistentes a tratamiento farmacológico, tales como la depresión severa, manías, esquizofrenia, delirium y síndrome neuroléptico maligno, entre otros (Tabla 1). Fue descrita inicialmente en el año 1938 por Hugo Cerletti siendo usada por mucho tiempo sin muchas bases fisiopatológicas y de manera rudimentaria, por lo que se asoció a importantes efectos adversos como fracturas óseas, confusión y trastornos de la memoria. Hoy en día la aplicación de esta terapia es mucho más segura gracias a los cambios tecnológicos en los equipos utilizados en la TEC, que entregan cantidades sustancialmente menores de estímulos eléctricos, junto a los cambios en las normas que regulan su aplicación, monitorización, el uso de anestesia y el entendimiento de las respuestas fisiológicas a la TEC.

En esta revisión veremos algunos aspectos generales sobre la TEC, sus indicaciones y la respuesta fisiológica que genera en el organismo. Junto con eso veremos las consideraciones que son importantes para el anestesista, desde la visita preanestésica, técnica utilizada, drogas disponibles y efectos adversos frecuentes.

\section{¿Qué es la TEC?}

La TEC es un procedimiento que consiste en la aplicación de pequeñas corrientes eléctricas para generar una descarga neuronal sincronizada que dure al menos 20-25 segundos, con el propósito de lograr la mejoría sintomática de diversas patologías. Pese a lo ampliamente usada en psiquiatría y a la mejoría de las técnicas y la práctica clínica de la TEC, los mecanismos por los cuales produce su efecto terapéutico aún son desconocidos. Entre las hipótesis existentes hay quienes afirman que habría cierta influencia sobre el sistema neurotransmisor, ya que se ha demostrado un aumento en la liberación de neurotransmisores de monoamina, particularmente dopamina y serotonina tanto en animales como en humanos ${ }^{1,2}$. Otra hipótesis se basa en el efecto que produce la TEC en el eje hipotálamohipófisis-suprarrenal, ya que se ha demostrado que las convulsiones repetidas aumentan la producción y liberación de un péptido hipotalámico que se relaciona con la mantención del estado de ánimo ${ }^{3}$. Diversos estudios indican que la TEC tiene un efecto trófico sobre el sistema nervioso central con la generación de nuevas neuronas en el hipocampo. Además se plantea que la TEC puede tener un rol con factores neurotrópicos, como el demostrado incremento del brain-derived neurotrofic factor (BDNF) $)^{4,5}$.

Sin embargo, a pesar del conocimiento cada vez mayor acerca de los efectos de la TEC sobre el sistema nervioso central, los mecanismos que explican la eficacia terapéutica en desórdenes psiquiátricos permanecen desconocidos.

\footnotetext{
Interno de Medicina. Pontificia Universidad Católica de Chile.

Residente de anestesiología. Pontificia Universidad Católica de Chile.

Profesor asistente. División de Anestesiología. Facultad de Medicina. Pontificia Universidad Católica de Chile.

Psiquiatra de adultos. Complejo Asistencial Sótero del Río.

Profesora asociada adjunta. División de Anestesiología. Facultad de Medicina. Pontifica Universidad Católica de Chile.
} 
Tabla 1. Indicaciones de terapia electroconvulsiva. (Adaptado de Baghai TC et al) ${ }^{11}$

\begin{tabular}{|c|c|}
\hline Categoría de indicación de TEC & Indicación \\
\hline \multirow[t]{6}{*}{ TEC como primera línea de tratamiento } & - Catatonía febril \\
\hline & - Síndrome neuroléptico maligno \\
\hline & - Episodio depresivo severo \\
\hline & - Psicosis esquizoafectiva \\
\hline & - Esquizofrenia \\
\hline & - Efectos de terapia psico-farmacológica intolerables o riesgosos \\
\hline \multirow[t]{6}{*}{ TEC como segunda línea de tratamiento } & Cuando el tratamiento médico falla en: \\
\hline & - Depresión \\
\hline & - Psicosis esquizoafectiva \\
\hline & - Esquizofrenia \\
\hline & - Manía \\
\hline & - Enfermedades orgánicas con síntomas depresivos o sicóticos \\
\hline \multirow[t]{6}{*}{ TEC es probablemente efectiva } & - Trastorno obsesivo-compulsivo resistente a tratamiento \\
\hline & - Disquinesias resistente a tratamiento \\
\hline & - Síndrome Gilles de la Tourette \\
\hline & - Epilepsia resistente a tratamiento \\
\hline & - Enfermedad de Parkinson resistente a tratamiento \\
\hline & - Estatus convulsivo refractario \\
\hline
\end{tabular}

\section{Respuesta fisiológica a la TEC}

Inicialmente el estímulo eléctrico causa una respuesta vagal en la cual suele ocurrir bradicardia e incluso asistolia de corta duración, generalmente previa a la convulsión. Mientras más prolongado es el estímulo eléctrico antes de que comience la convulsión, más probable es que la bradicardia o la asistolia ocurran. Además, esta fase podría ser más prolongada en aquellos pacientes en tratamiento con $\beta$ bloqueadores. Por tanto, es recomendable utilizar premedicación anticolinérgica en los pacientes sometidos a TEC .

Una vez que comienza la convulsión, existe una descarga simpática caracterizada por un aumento en la frecuencia cardiaca en un $20 \%$ o más y un aumento en la presión sanguínea en un 30-40\% en relación a sus valores basales, lo cual genera un aumento del consumo de oxígeno miocárdico ${ }^{7}$. Además, durante este período puede existir un incremento del flujo sanguíneo cerebral, de la presión intracraneana, la presión intraocular y la presión intragástrica ${ }^{8,9}$. Se ha descrito que la respuesta hiperdinámica puede llegar a producir isquemia e infarto miocárdico, alteraciones vasculares neurológicas y pérdidas de memoria a corto plazo que pueden llegar a serias disfunciones cognitivas en casos más extremos ${ }^{10}$.

\section{Indicaciones y contraindicaciones de la TEC}

La indicación de la TEC debe ser una decisión que no sólo se base en el diagnóstico del paciente sino que también en base a los antecedentes personales, respuesta a tratamientos previos y las condiciones del servicio ${ }^{11}$. Es por esto que actualmente entre los psiquiatras existen variadas opiniones acerca de las indicaciones de TEC. De manera general, podemos establecer un grupo en el cual la TEC es considerada como primera línea de tratamiento, otro en la que la TEC es utilizada frente al fracaso de otras terapias, principalmente farmacológica, y un grupo de patologías en el que no existen ensayos clínicos aleatorizados que demuestren la eficacia y utilidad de la terapia, pero en las que existen reportes de casos o series en las que han existido buenos resultados (Tabla 1).

Actualmente no existen contraindicaciones absolutas para la aplicación de la terapia, sin embargo, existen situaciones de mayor cuidado que para el psiquiatra y el anestesista son importantes de conocer, con objeto de sopesar los riesgos y beneficios de la intervención y así tomar la mejor decisión en conjunto con el paciente y/o su familia. Las contraindicaciones relativas se pueden observar en la Tabla 2. 
Tabla 2. Contraindicaciones relativas de terapia electroconvulsiva. (Adaptado de Baghai TC et al ${ }^{11}$ )

\begin{tabular}{|ll|}
\hline Categoría & Condición clínica \\
Aumento de presión intracraneana & Al momento de la terapia \\
Infarto cerebral & Menor a 3 meses \\
Infarto al miocardio & Menor a 3 meses \\
Tumor intracerebral & Incluyendo edema cerebral \\
Trastornos cardiovasculares & $\begin{array}{l}\text { Arritmias cardiacas, enfermedad coronaria, insuficiencia } \\
\text { cardiaca, valvulopatías, aneurisma de la aorta }\end{array}$ \\
Trastornos médicos & $\begin{array}{l}\text { Alteraciones de la coagulación, enfermedad hepática } \\
\text { severa, enfermedad pulmonar severa, feocromocitoma, } \\
\text { desprendimiento de retina, glaucoma }\end{array}$ \\
Trastornos neurológicos & $\begin{array}{l}\text { Sangrado intra-craneal, malformaciones vasculares intra- } \\
\text { cerebrales, isquemia cerebral, inflamación cerebral, hi- } \\
\text { drocefalia, demencias, enfermedades de ganglios basales, } \\
\text { craneotomías, trauma cerebral severo }\end{array}$ \\
Trastornos ortopédicos & Osteoporosis \\
\hline Hernia esofágica & Riesgo de aspiración aumentado. Considerar intubación \\
\hline
\end{tabular}

\section{Consideraciones anestésicas en TEC}

\section{Visita pre-anestésica:}

Con el objetivo de planificar el procedimiento a realizar, el anestesiólogo debe evaluar al paciente para determinar la indicación de la TEC y la condición médica en la que éste se encuentra, especialmente aquellas que puedan estar asociadas a un incremento del riesgo de los efectos adversos de la TEC. El paciente debe estar enterado del procedimiento al que se someterá y haber firmado el consentimiento escrito para ello. Sólo en caso de que el paciente no esté capacitado para esto, el consentimiento debe ser firmado por un familiar o representante legal, conforme a la ley y a las políticas de cada institución.

En general el efecto terapéutico del tratamiento requiere de varias sesiones. Es por eso que es recomendable una evaluación formal al comienzo de la serie, y que ésta sea registrada de manera óptima para contar con esta información en las sesiones futuras. Además, la historia se debe actualizar en cada sesión con las características del tratamiento realizado, drogas utilizadas y eventos importantes que sucedan durante la terapia, como bradicardia, asistolia o crisis hipertensivas, que pueden justificar cambios en el manejo anestésico a futuro.

Con respecto a las comorbilidades, son de especial atención los antecedentes de enfermedad cardiopulmonar y la historia de cirugías previas, incluyendo tipo de anestesia utilizada y complicaciones, principalmente dificultad en la ventilación y/o intubación, parálisis prolongada o reacción ad- versa a medicamentos ${ }^{12}$. Es necesario un apropiado examen físico y evaluación de laboratorio según los antecedentes obtenidos en la visita preanestésica. Por ser un procedimiento bajo anestesia general, se hace necesario que el paciente cumpla con ayuno.

Dado que el estándar de bloqueador neuromuscular utilizado es la succinilcolina, se debe preguntar dirigidamente si existe historia personal o familiar de hipertermia maligna o miopatías asociadas a elevación de creatinfosfokinasa.

\section{Laboratorio e imágenes:}

Como regla general no se requiere ningún test de laboratorio específico. Sin embargo, a modo de recomendación, se sugiere contar con electrolitos plasmáticos en pacientes que estén recibiendo diuréticos o padezcan alguna patología que pudiera producir su alteración, como enfermedad renal crónica, por su riesgo de hipercalemia, y con electrocardiograma en mayores de 50 años, pacientes con patología cardiovascular y en aquellos en los que se sospeche prolongación del espacio QT, en quienes aumenta el riesgo de eventos cardiacos ${ }^{13}$.

En pacientes con historia sugerente de patología coronaria es necesaria una evaluación cardiológica y un test diagnóstico de isquemia. Las neuroimágenes son necesarias en pacientes en quienes haya sospecha de aumento de la presión intracraneana (tumor intracraneano, AVE, encefalitis, etc.) o sospecha de causa orgánica responsable de la patología por la que se decide la TEC ${ }^{14}$. En general se solicita que todos los pacientes cuenten con un 
electroencefalograma, ya que éste se ve alterado posterior a la terapia.

\section{Fármacos:}

Como a todo paciente que se somete a un procedimiento donde vaya a requerir de anestesia general se deben evaluar los medicamentos que está recibiendo. Las drogas de uso habitual en psiquiatría tales como antidepresivos, antisicóticos y litio pudiesen tener efectos sinérgicos. Sin embargo, por sus resultados dispares, en la práctica habitual se prefiere disminuirlas al mínimo posible ${ }^{15,16}$. Por otro lado, las benzodiacepinas pudiesen interferir con la TEC dado sus propiedades que pueden llevar a un aumento del umbral convulsivante, acortamiento de la duración de las convulsiones y, en su defecto, disminución de la eficacia clínica de la TEC, por lo que se debe evitar su uso o disminuirlo al mínimo con drogas de vida media corta ${ }^{17}$. El uso de los anticonvulsivantes podrían disminuir la eficacia de la TEC, por eso cuando son utilizados para la estabilización del ánimo deberían ser reducidos y discontinuados previo a la terapia, pero mantenidos en aquellos pacientes que están indicados por epilepsia ${ }^{18}$. Medicamentos cardiovasculares como antihipertensivos (no diuréticos) y antianginosos, deben ser administrados como de costumbre hasta 2 horas antes del procedimiento. Se deben evitar diuréticos por el riesgo de relajación de esfínter durante la convulsión ${ }^{19}$. Medicamentos antiasmáticos como corticoides y beta agonistas inhalatorios, pueden ser administrados de forma normal de ser necesarios para evitar la broncoconstricción ${ }^{19}$. Se debe evitar el uso de teofilina pues se ha asociado a convulsiones prolongadas y status epiléptico ${ }^{20}$. Medicamentos gastrointestinales como inhibidores de la bomba de protones o anti $\mathrm{H}_{2}$ deben administrarse en pacientes con tratamiento de reflujo gastroesofágico para minimizar el riesgo de aspiración ${ }^{12,19}$. No existe evidencia clara actual acerca del efecto de la TEC sobre los niveles de azúcar y el rol que juegan los hipoglucemiantes orales, por eso se recomienda mantener estos medicamentos previo al procedimiento $^{19}$.

\section{Técnica anestésica:}

Los objetivos esenciales de la anestesia para TEC son una pérdida rápida de conciencia, una atenuación efectiva de la respuesta hiperdinámica al estímulo eléctrico, evitar los movimientos del paciente y una recuperación pronta de la ventilación espontánea y de la conciencia. El logro de los objetivos anteriores no debe afectar la actividad convulsiva.

Todos los pacientes deben ser monitorizados con electrocardiograma, oxímetro de pulso y presión arterial no invasiva. La monitorización de la ventilación no necesariamente requiere capnografía, pero ésta puede ser útil en pacientes obesos o con apnea del sueño ${ }^{21}$. El monitoreo con BIS ha sido usado con resultados discordantes. Sin embargo, se debe utilizar un monitor de encefalograma, para que el psiquiatra pueda verificar si se produce una descarga neuronal sincrónica y medir de manera precisa su duración ${ }^{22}$.

Se debe verificar el correcto funcionamiento de la máquina de anestesia. El equipamiento apropiado para resucitación debe estar disponible, así como un laringoscopio, tubo traqueal y máscara laríngea para tratar una emergencia en el manejo de la vía aérea ${ }^{8}$ o un paro cardiaco. Se debe asegurar una vía venosa permeable que permanecerá durante todo el tiempo que el paciente se encuentre en el área del procedimiento y recuperación, y se retirará al momento del alta en los pacientes ambulatorios. En nuestra institución, luego de realizada la TEC, también se debe retirar la vía venosa en los pacientes hospitalizados en el Servicio de Salud Mental.

El paciente debe ser preoxigenado con mascarilla facial previo al procedimiento. Después de esto se administra el fármaco de inducción. En la sesión inicial se debe titular la dosis de agente de inducción, teniendo en cuenta que se debe usar la dosis más baja que produzca hipnosis para facilitar una convulsión adecuada. Luego que el paciente está dormido se administra el relajante muscular. Para la medición de la relajación muscular se debe utilizar un estimulador de nervio o buscar la abolición del reflejo de Babinski ${ }^{22}$.

Una vez que el paciente cae en apnea se debe hiperventilar a presión positiva por uno a dos minutos, ya que esto disminuye los niveles sanguíneos de $\mathrm{CO}_{2}$ y disminuye el umbral convulsivante, facilitando que se produzca la convulsión e incrementando la duración de ésta ${ }^{12}$. Sólo se interrumpe la ventilación para aplicar el estímulo eléctrico y rara vez es necesario intubar al paciente. Previo a la descarga eléctrica se debe introducir un dispositivo protector flexible para proteger los dientes, labios y lengua. El procedimiento debe coordinarse con el psiquiatra, quien instalará las paletas de descarga al paciente y aplicará el estímulo eléctrico. El mentón del paciente debe sostenerse durante el paso de la corriente del estímulo para mantener la mandíbula firmemente contra el protector bucal.

\section{Drogas utilizadas en la TEC:}

Es indispensable saber manejar la particular interacción existente entre la anestesia y la TEC, ya que si la anestesia resulta ser demasiado superfi- 
cial, la pérdida de conciencia puede ser incompleta y la reacción autonómica puede ser muy intensa. Por otro lado, si la anestesia es demasiado profunda puede elevar el umbral de la crisis con lo cual se puede comprometer la eficacia terapéutica.

\section{a. Premedicación:}

La TEC genera una descarga parasimpática inicial y posteriormente una descarga simpática que es mucho más duradera, lo que genera cambios hemodinámicos en el paciente.

Para prevenir la hipertensión y la taquicardia se ha planteado el uso de diversos fármacos que veremos a continuación:

- $\beta$-bloqueadores: Se ha estudiado el uso de $\beta$ bloqueadores de acción corta como esmolol y labetalol. No hay dudas sobre el tratamiento en los cambios hemodinámicos que ocurre después del procedimiento con este tipo de drogas, pero su uso profiláctico para reducir el riesgo aún resulta controversial ya que el riesgo general de complicaciones cardiovasculares debido a la terapia es bajo y no hay estudios que demuestren que el riesgo de complicaciones cardiacas de la TEC disminuya ${ }^{23,24}$. Por otro lado, la Asociación Americana de Psiquiatría el año 2001 , señaló que los $\beta$-bloqueadores profilácticos podrían incrementar el riesgo de asistolia ${ }^{25}$, que el aumento absoluto de la presión arterial y frecuencia cardiaca no es mayor en personas normales versus personas con cifras basalmente elevadas y que la mitigación de la respuesta hemodinámica podría asociarse a reducción del aporte de $\mathrm{O}_{2}$, además de disminuir la duración de la convulsión y efectividad de la TEC ${ }^{26-28}$. Finalmente, sólo se plantea el uso de estos medicamentos en pacientes con hipertensión inestable previo al inicio de la TEC y en aquellos con alto riesgo de complicación por cifras tensionales elevadas, como pacientes con aneurisma intracraneano, angina inestable e IAM reciente ${ }^{14}$. Las dosis recomendadas son: labetalol 5-20 mg intravenoso (IV) o esmolol 10-50 mg IV.

- Bloqueadores de canales de calcio: Se ha estudiado el uso de nifedipino y nicardipino para el manejo de la respuesta hiperdinámica. En pacientes de mayor edad que no responden satisfactoriamente al uso de labetalol, la utilización de labetalol más nifedipino ha mostrado ser segura y efectiva ${ }^{29}$. Además, la utilización de nifedipino $10 \mathrm{mg}$ sublingual 20 minutos previos a la TEC, ha mostrado atenuar el incremento en la presión arterial media ${ }^{30}$. Estudios más recientes avalan el uso de nicardipino en dosis de 40 $\mu \mathrm{g} \cdot \mathrm{kg}^{-1}$ IV inmediatamente previo al estímulo eléctrico para controlar la hipertensión arterial, pero que en dosis mayores se asocia a un rápido aumento de la frecuencia cardiaca ${ }^{31}$.

- a2-agonista/antagonista: Clonidina oral en dosis de $0,05 \mathrm{mg}$ a $0,3 \mathrm{mg}, 60$ a 90 minutos previo a la terapia, ha mostrado disminuir la presión arterial media previo al estímulo, sin embargo, no ha mostrado cambios significativos en el aumento de la frecuencia cardiaca y de la presión arterial después del estímulo ${ }^{32}$. Dexmedetomidina en dosis $1 \mu \mathrm{g} \cdot \mathrm{kg}^{-1} \mathrm{IV}$ ha mostrado ser efectiva en el manejo de la respuesta hiperdinámi$\mathrm{ca}^{33,34}$.

- Vasodilatadores de acción directa: El uso de nitroglicerina en dosis $3 \mu \mathrm{g} \cdot \mathrm{kg}^{-1}$ IV previo a la TEC ha mostrado variables hemodinámicas significativamente más bajas que con el uso de esmolol, sin generar cambios en la duración de la convulsión ${ }^{35}$. Además, el uso de nitroglicerina $0,4 \mathrm{mg}$ sublingual previo a la terapia también ha mostrado disminuir efectivamente la respuesta hiperdinámica ${ }^{36}$. El uso de nitroglicerina además inhibe el incremento en la presión intracraneana.

Los efectos parasimpáticos de la TEC son: aumento de la salivación, bradicardia transitoria e incluso asistolia. Para su prevención se ha recomendado:

- Atropina: En dosis 0,2-0,5 mg IV, 3 a 5 minutos previos al procedimiento, ha demostrado disminuir la incidencia de bradicardias, pero aumentar la ocurrencia de taquicardias ocurridas después de la $\mathrm{TEC}^{37}$. La droga tiene el efecto adicional de disminuir la salivación y las secreciones de la vía aérea.

- Glicopirrolato: Es una droga anticolinérgica que carece de actividad en el sistema nervioso central por lo que tiene la ventaja de producir menos taquicardia después de la TEC, manteniendo las otras ventajas del bloqueo colinérgico, lo cual lo transforma en la droga de elección. Estudios más recientes muestran además, que la droga puede atenuar los efectos de la oxigenación y perfusión cerebral de la TEC ${ }^{38}$. Se utiliza en dosis de 0,1 a $0,3 \mathrm{mg} \mathrm{IV}$.

\section{b. Inductores:}

La eficacia de la TEC está directamente relacionada con el tiempo de convulsión que se logra ${ }^{8}$. Dado que muchas drogas anestésicas usadas tienen propiedades anticonvulsivantes podrían interferir en la duración de la convulsión de una forma dosis dependiente y por ende en la efectividad del tratamiento, por lo que es necesario su uso adecuado y 
siempre hacer un balance entre la anestesia usada y la capacidad de generar una convulsión exitosa.

El objetivo de la anestesia general en TEC, como ya se señaló, es reducir el impacto psicológico y fisiológico inducido por el estrés, sin llegar a una anestesia profunda que pueda elevar el umbral convulsivo y comprometer la eficacia de la terapia. Es por eso que los inductores se utilizan en dosis subhipnóticas y no en dosis anestésicas habituales.

- Methohexital: Es la droga de elección y más ampliamente usada como anestésico en TEC, por lo que es considerada el gold estándar. Las dosis recomendadas son $0,75-0,9 \mathrm{mg} \cdot \mathrm{kg}^{-1} \mathrm{se}-$ gún la Royal College of Psychiatrists y 0,75-1,0 $\mathrm{mg} \cdot \mathrm{kg}^{-1}$ según la American Psychiatric Association, aunque el consumo crónico de sustancias como el alcohol y las benzodiacepinas pudiesen determinar dosis anestésicas mayores ${ }^{8}$. El methohexital tiene poco efecto sobre el umbral convulsivante y, por tanto, es el inductor con menor efecto anticonvulsivante ${ }^{37}$. En comparación con propofol, se generan convulsiones más largas y cambios hemodinámicos más modera$\operatorname{dos}^{39}$. No se debe usar cuando hay contraindicación a barbitúricos (ejemplo, porfiria intermitente aguda). Esta droga no está disponible en Chile.

- Propofol: Tiene los efectos anticonvulsivantes más potentes de las drogas anestésicas usadas en TEC, pero el uso de dosis hipnóticas mínimas $\left(0,75 \mathrm{mg} \cdot \mathrm{kg}^{-1}\right)$ se asocian a duración de la convulsión comparables a aquella del gold estándar, llegando incluso a convulsiones clínicamente aceptables con dosis mayores $(1,5$ $\left.\mathrm{mg} \cdot \mathrm{kg}^{-1}\right)^{40,41}$. Dado los efectos depresores cardiovasculares conocidos del propofol, reduce la respuesta hemodinámica aguda durante la $\mathrm{TEC}^{42}$. Vaidya et al. muestran que el propofol, en comparación con methohexital, no afecta la mejoría en los síntomas depresivos, la duración de la estadía hospitalaria ni la recuperación cognitiva, sin embargo, en terapia con electrodo unilateral los pacientes que recibieron propofol necesitaron más sesiones de TEC que los que recibieron methohexital y en los pacientes con terapia con dos electrodos requirieron dosis mayores de inducción y estímulos eléctricos más altos $^{43}$. La eficacia comparable con la droga estándar, su corta vida media, el despertar placentero de los pacientes y su efecto antiemético la hacen la droga de elección en TEC ambulatoria en nuestro país. El propofol ha sido usado en combinación con dexmedetomidina, lo que generó frecuencia cardiaca y presión arterial más baja posteriores a la TEC, sin cambiar la dura- ción de la convulsión ni el tiempo de recuperación $^{34}$.

- Tiopental: Acorta la duración de la convulsión en el EEG comparado con methohexital y además se ha asociado a bradicardia sinusal y contracción ventricular prematura ${ }^{37}$. Aumenta más que el propofol la velocidad del flujo de la arteria cerebral media medida inmediatamente después de la TEC, razones por la cual no es mayormente recomendado ${ }^{44}$. Tiene un perfil hemodinámico similar al etomidato y al propofol. Pese a eso, es una droga utilizada en Chile y muchos lugares de Europa y Asia dada su rápida desaparición de efectos, su vida media intermedia y la amplia experiencia por parte de algunos anestesistas. Las dosis recomendadas son $1,5-2,5 \mathrm{mg} \cdot \mathrm{kg}^{-1}$.

- Etomidato: En comparación con las drogas anteriores se asocia a mayor duración de la convulsión y podría ser de ayuda en pacientes con tiempos convulsivos breves (menores a 20 segundos), en pacientes refractarios o que no convulsionan ${ }^{45}$. Inclusive existen casos en que ha generado convulsiones previo a la aplicación del estímulo. Por su tendencia a mantener la presión arterial, en combinación con la descarga simpática asociada, puede manifestar una marcada hipertensión en comparación con los barbitúricos y el propofol. Las dosis utilizadas son $0,15-0,3 \mathrm{mg} \cdot \mathrm{kg}^{-1}$.

- Ketamina: es un anestésico intravenoso con propiedades sedativas y analgésicas que ha sido utilizada como agente inductor en TEC. Dado sus propiedades simpaticomiméticas, las variables hemodinámicas son comparativamente mayores a otras drogas por lo que es menos recomendada que el methohexital y el propofol ${ }^{8}$. Además, disminuye la duración de la convulsión al EEG, aún con dosis menores. El riesgo teórico de generar agitaciones y alucinaciones la hacen una alternativa menos atractiva.

- Sevoflurano: Como agente de inducción inhalatoria, en comparación con propofol, muestra tiempos de convulsión más cortos sin alterar su calidad $^{46}$. En comparación con propofol y tiopental genera mayores incrementos hemodinámicos postictales y tiene frecuencia de eventos adversos comparables. En la actualidad puede considerarse una opción anestésica en TEC.

\section{c. Relajantes musculares:}

El uso de relajación muscular atiende al hecho de evitar las complicaciones traumáticas que se pueden producir por la intensa contracción muscular, durante la fase tónica de la convulsión, ta- 
les como fracturas, esguinces, luxaciones y aplastamientos vertebrales. Así también, su utilización atenúa el impacto fisiológico de la crisis, producto de que la contracción muscular intensa genera aumento del consumo de $\mathrm{O}_{2}$ y la producción de $\mathrm{CO}_{2}$ con el consiguiente aumento del $\mathrm{K}^{+}$plasmático, acentúa la respuesta endocrina al estrés y su impacto cardiovascular. Debido a esto, se debe utilizar una técnica especial para observar y medir la duración de la manifestación motora de la convulsión. Previo a la inyección de relajante muscular se infla un manguito de presión (o manguito de isquemia) por sobre la presión sistólica del paciente en una de las extremidades inferiores (en TEC unilateral debe usarse la extremidad inferior del hemicuerpo ipsilateral) la cual no sufrirá el efecto de la relajación muscular, por lo que mediante la aplicación de los electrodos será posible medir la convulsión motora. - Succinilcolina: Es el relajante muscular habitualmente utilizado. Dosis de 0,3 a $0,5 \mathrm{mg} \cdot \mathrm{kg}^{-1}$ producen un $95 \%$ de bloqueo en el músculo abductor del pulgar, sin embargo, en la práctica clínica habitual se recomienda utilizar 0,75-1 $\mathrm{mg} \cdot \mathrm{kg}^{-1}$, debido principalmente a la gran variabilidad interindividual del efecto. Tiene un inicio de acción entre 30 a 60 segundos y su efecto dura entre 5 a 10 minutos, dependiendo de la dosis utilizada ${ }^{47}$. Incluso pequeñas dosis de este relajante pueden producir efectos adversos como mialgias, hipertermia o hipercalemia en pacientes con factores de riesgo como historia familiar de hipertermia maligna, síndrome neuroléptico maligno, esquizofrenia catatónica e intoxicación por órganofosforados. Es en estos casos en los que se deben utilizar relajantes musculares no depolarizantes.

- Rocuronio: En dosis de $0,3 \mathrm{mg} \cdot \mathrm{kg}^{-1}$, es apropiado y efectivo en aquellos casos en que está contra-indicado el uso de succinilcolina y además genera un perfil de relajación muscular parecido a succinilcolina en cuanto a inicio de la acción y duración del efecto. Dosis mayores generan un inicio de la acción más rápido, pero con mayor tiempo de duración. Su antagonista específico, sugamadex, presenta la ventaja de poder ser usado en cualquier momento, independiente del grado de relajación muscular presente y con pocos efectos adversos asociados. Lamentablemente su alto costo es una limitante en Chile para un tratamiento que requiere de varias sesiones.

- Mivacurio: En dosis de $0,2 \mathrm{mg} \cdot \mathrm{kg}^{-1}$ administrado 3 minutos antes de la TEC es también una buena alternativa por su corta duración de acción, cuyo efecto puede ser revertido con neostigmina, o puede esperarse el cese espontáneo del efecto cuando la neostigmina no está recomendada ${ }^{48}$. Es necesario señalar que el mivacurio es metabolizado por pseudocolinesterasas, por lo que, igual que la succinilcolina, no es recomendado en pacientes en los cuáles se sospecha deficiencia de ellas.

\section{Efectos adversos}

La TEC es una terapia muy bien tolerada y con un bajo riesgo de complicaciones severas. La mortalidad varía entre 1:50.000 y 1:25.000 mientras que las complicaciones severas se ven en 1:10.000 $\operatorname{casos}^{48}$.

Los efectos adversos descritos se pueden dividir en efectos médicos y cognitivos.

Dentro de los efectos médicos los más frecuentes son cefalea, náuseas y vómitos. Hasta un $45 \%$ de los pacientes presenta cefalea, la que puede ser manejada con analgésicos como paracetamol o antiinflamatorios no esteroidales. Para pacientes que sufren previamente de migraña se ha descrito el uso de triptanes ${ }^{11}$. Las náuseas pueden ser manejadas con metoclopramida $u$ ondansetron. Los eventos cardiovasculares como isquemia cardiaca, IAM, arritmias y emergencia hipertensiva han sido descritos, pero son poco habituales. En algunas ocasiones se han descrito convulsiones prolongadas por más de 60 a 180 segundos; en estos casos, se debe considerar el uso de un anticonvulsivante como benzodiacepinas o un inductor anestésico. En casos de relajación muscular prolongada se debe mantener la asistencia respiratoria y la monitorización de la oxigenación. El dolor muscular es raro y se previene con la adecuada relajación muscular. En los pacientes que sufren trastorno bipolar la TEC puede generar un viraje a manía o hipomanía ${ }^{49}$. El uso de litio y otros estabilizadores del ánimo pueden reducir este riesgo significativamente.

Los pacientes pueden presentar agitación psicomotora posterior a la convulsión, que en general se resuelve sin medidas farmacológicas, pero que en casos más severos o persistentes pueden ser tratados con lorazepam o propofol endovenoso y medidas de contención física en caso de ser necesario.

Los efectos adversos cognitivos pueden afectar entre un 51 a $79 \%$ de los pacientes sometidos a TEC, dependiendo de la dosis y la aplicación del estímulo eléctrico ${ }^{50}$. Un estado confusional agudo se puede presentar como resultado de la convulsión y de la anestesia. Generalmente éste se resuelve entre 10 a 30 minutos posterior a la convulsión. El estado confusional agudo puede ser mayor en pa- 
cientes más añosos, según la dosis y tipo de droga inductora y la medicación sistémica y psicotrópica que esté recibiendo ${ }^{11}$.

La amnesia anterógrada es una capacidad disminuida para retener nueva información adquirida posterior a la terapia. Ésta puede ocurrir durante la serie de TEC y generalmente regresa a su estado basal dos semanas después de completar la terapia. La amnesia retrógrada implica olvidar recuerdos previos a la TEC. La memoria impersonal se ve más afectada que la personal y la amnesia retrógrada se recupera más lentamente que la anterógrada ${ }^{51}$.

Se ha descrito que entre un 29 a $55 \%$ de los pacientes presentan pérdida de memoria persistente o permanente, cuya etiología es controversial ${ }^{51}$. De todos modos, el paciente que se somete a TEC generalmente presenta síntomas depresivos severos, por lo que parece razonable tolerar algunos efectos adversos en pos de resolver el episodio depresivo.

En datos de nuestro país, los efectos adversos se presentaron en $3,8 \%$ de los tratamientos de una serie de la Pontificia Universidad Católica de Chile publicada en $2007^{52}$. Además el carácter leve de las complicaciones hace que actualmente se considere un procedimiento seguro.

\section{CONCLUSIONES}

La TEC es un procedimiento ampliamente usado en la psiquiatría como una terapia eficaz para patologías graves resistentes a tratamiento médico. Inicialmente se asoció a muchos mitos, pero hoy en día significa una posibilidad real de mejorar depresiones graves, manías, trastorno obsesivo compulsivo, esquizofrenia e incluso algunas patologías médicas como el síndrome neuroléptico maligno, entre otras. La incorporación de la anestesia ha permitido grandes avances en términos de seguridad y tolerancia a la terapia.

Durante una sesión de TEC se realizan dos procedimientos esenciales: por una parte se anestesia al paciente y por otra se aplica el electroshock propiamente tal. En nuestra experiencia, estas tareas, si bien están relacionadas y son secuenciales, deben ser realizadas en forma independendiente por un(a) anestesista y un(a) psiquiatra respectivamente. El psiquiatra evalúa al paciente antes de cada sesión, programa la máquina de $\mathrm{TEC}$, determina las características del estímulo para mayor efectividad y aplica la descarga eléctrica. El anestesista se preocupa exclusivamente de las tareas propias de la anestesia general, de proteger los dientes y mucosas orales, de tratar las manifestaciones car- diovasculares de la TEC cuando corresponda y del adecuado despertar del paciente. Este trabajo en equipo permite realizar la TEC ajustada a las caraterísticas de cada paciente y aumenta los beneficios y la seguridad del procedimiento, por lo que es recomendable que ésta sea la norma de todos los servicios.

Como todo procedimiento con uso de anestesia se debe realizar una adecuada evaluación previa a la TEC, enfocándose en los antecedentes médicos que puedan significar un riesgo mayor, así como también en los fármacos que puedan afectar la actividad convulsiva, para así tomar la precauciones necesarias y pedir las evaluaciones imagenológicas o de laboratorio pertinentes, del mismo modo se debe estar preparado para los principales efectos adversos que pudiesen desencadenarse durante la TEC.

Existen algunos hechos prácticos que hemos aprendido en el camino (más de 10 años) y que nos parece importante resaltar, en especial cuando la anestesia para TEC no forma parte de los programas de formación actuales. Es fundamental obtener el consentimiento informado escrito de anestesia en todo paciente, sin excepción; de la misma manera, es trascendental la comunicación permanente y fluida con el psiquiatra tratante. Si bien la TEC es un procedimiento ambulatorio y seguro, se debe realizar en un área que disponga de máquina de anestesia, monitorización estándar y sala de recuperación. En nuestra institución realizamos estos procedimientos en el pabellón quirúrgico, con monitorización continua lo que nos ha permitido diagnosticar eventos cardiovasculares oportunamente ${ }^{52}$. Las dosis de anestésicos se determinan en las primeras sesiones, de acuerdo a la respuesta de cada paciente; habitualmente utilizamos 1 a $1,5 \mathrm{mg} \cdot \mathrm{kg}^{-1}$ de propofol y $1 \mathrm{mg} \cdot \mathrm{kg}^{-1}$ de succcinilcolina sin precurarización, con lo cual obtenemos buenos niveles de sedación, óptima relajación muscular y convulsiones adecuadas. Por lo anterior, es importante registrar cada sesión de TEC en hoja ad-hoc, que señale las dosis de anestésicos utilizadas, las variables hemodinámicas y respiratorias, la duración de las convulsiones y cualquier evento no deseado; en cada sesión de TEC debe estar disponible el registro de las sesiones anteriores.

Finalmente, es importante que los anestesistas incorporen en términos generales el conocimiento de estas patologías psiquiátricas, el funcionamiento de la TEC, sus indicaciones más importantes y en especial los pilares de su manejo anestésico que como vimos difiere en gran medida de la anestesia general para otros procedimientos. 


\section{REFERENCIAS}

1. Rudorfer MV, Risby ED, Hsiao JK, et al. Disparate Biochemical Actions of Electroconvulsive Therapy and Antidepressant Drugs. Convuls Ther 1988; 4: 133-140.

2. Nikisch G, Mathé AA. CSF monoamine metabolites and neuropeptides in depressed patients before and after electroconvulsive therapy. Eur Psychiatry 2008; 23: 356-359.

3. Fink M, Nemeroff CB. A neuroendocrine View of ECT. Convuls Ther 1989; 5: 296.

4. Madsen T, Treschow A, Bengzon $\mathrm{J}$, et al. Increased neurogenesis in a model of electroconvulsive therapy. Biol Psychiatry 2000; 47: 10431049.

5. Okamoto T, Yoshimura R, Ikenouchi-Sugita A, et al. Efficacy of electroconvulsive therapy is associated with changing blood levels of homovanillic acid and brain-derived neurotrophic factor (BDNF) in refractory depressed patients: a pilot study. Biol Psychiatry 2008; 32: 1185-1190.

6. Deiine S, Frost E. Electroconvulsive therapy and anesthesia. Int Anesthesiol Clin 2009; 47: 81-92.

7. Wells D, Davies G. Hemodynamic changes associated with electroconvulsive therapy. Anesth Analg 1987; 66: 1193-1195.

8. Zhengnian D, Paul F. Anesthesia for Electroconvulsive Therapy. Anesth Analg 2002; 94: 13511364.

9. Saito S, Miyoshi S, Yoshikawa D, et al. Regional cerebral oxygen saturation during electroconvulsive therapy: monitoring by nearinfrared spectrophotometry. Anesth Analg 1996; 83: 726-730.

10. López D, Sánchez M, V. Cobo J, et al. Infarto agudo de miocardio tras terapia electroconvulsiva. Rev Esp Cardiol 1999; 52: 536-538.

11. Baghai T, Möller H. Electroconvulsive therapy and its different indications. Dialogues Clin Neurosci 2008; 10: 105-117.

12. Kellner C, Bryson E. Anesthesia advances add to safety of ECT. Psychiatric Times 1998; 29: 16-18.

13. Pullen S, Rasmussen K, Angstman ER. The safety of electroconvulsive therapy in patients with prolonged QTc intervals on the electrocardiogram. J ECT 2011; 27: 192-200.

14. Tess AV, Smetana GW. Medical evaluation of patients undergoing electroconvulsive therapy. N Engl J Med 2009; 360: 1437-1444.

15. Sackeim H, Dillingham E, Prudic
J, et al. Effect of concomitant pharmacotherapy on electroconvulsive therapy outcomes: short-term efficacy and adverse effects. Arch Gen Psychiatry 2009; 66: 729-737.

16. Dolenc TJ, Rasmussen KG. The safety of electroconvulsive therapy and lithium in combination: a case series and review of the literature. $\mathrm{J}$ ECT 2005; 21: 165.

17. Greenberg RM, Pettinati HM. Benzodiazepines and Electroconvulsive Therapy. Convuls Ther 1993; 9: 262-273.

18. Sienaert P, Peuskens J. Anticonvulsants during electroconvulsive therapy: review and recommendations. J ECT 2007; 23:120.

19. Kellner C. Overview of electroconvulsive therapy (ECT) for adults. En UpToDate, Roy-Byrne (Ed). (Consultado el 03/04/2014).

20. Rasmussen KG, Zorumski CF. Electroconvulsive therapy in patients taking theophylline. J Clin Psychiatry 1993; 54: 427-431.

21. Gross JB, Bachenberg KL, Benumof JL. Practice guidelines for perioperative management of patients with obstructive sleep apnea. Anesthesiology 2006; 104: 10811095.

22. Rose C. Anesthetic and System Based Considerations for Electroconvulsive Therapy. ASA Refresher Courses in Anesthesiology 2008; 36(1): 143-154.

23. Castelli I, Steiner L, Kaufmann $\mathrm{M}$, et al. Comparative effects of esmolol and labetalol to attenuate hyperdynamic states after electroconvulsive therapy. Anesth Analg 1995; 80: 557-561.

24. Kovac A, Goto H, Arakawa K, et al. Esmolol bolus and infusion attenuates increases in blood pressure and heart rate during electroconvulsive therapy. Can J Anaesth 1990; 37: 58-62.

25. Burd J, Kettl P. Incidence of asystole in electroconvulsive therapy in elderly patients. Am J Geriatr Psychiatry 1998; 6: 203-211.

26. Webb MC, Coffey CE, Saunders WR, et al. Cardiovascular response to unilateral electroconvulsive therapy. Biol Psychiatry 1990; 28: 758-766.

27. Prudic J, Sackeim HA, Decina P, et al. Acute effects of ECT on cardiovascular functioning: relations to patient and treatment variables. Acta Psychiatr Scand 1987; 75: 344-351.

28. Howie MB, Black HA, Zvara D, et al. Esmolol reduces autonomic hypersensitivity and length of sei- zures induced by electroconvulsive therapy. Anesth Analg 1990; 71: 384-388.

29. Figiel G, De Leo B, Zorumski C, et al. Combined use of labetalol and nifedipine in controlling the cardiovascular response from ECT. J Geriatr Psychiatry Neurol 1993; 6: 20-24.

30. Wells DG, Davies GG, Rosewarne F. Attenuation of electroconvulsive therapy induced hypertension with sublingual nifedipine. Anaesth Intensive Care 1989; 17: 31-33.

31. Zhang Y, White P, Thornton L, et al. The use of nicardipine for electroconvulsive therapy: a doseranging study. Anesth Analg 2005; 100: 378-381.

32. Fu W, Stool LA, White PF, et al. Is oral clonidine effective in modifying the acute hemodynamic response during electroconvulsive therapy? Anesth Analg 1998; 86: 1127-1130.

33. Aydogan M, Yucel A, Begec Z, et al. The hemodynamic effects of dexmetomidine and esmolol in electroconvulsive therapy: a retrospective comparison. J ECT 2013; 29: 308-311.

34. Begec Z, Toprak H, Demirbilek S, et al. Dexmetomidine blunts acute hyperdinamic responses to electroconvulsive therapy without altering seizure duration. Acta Anaesthesiol Scand 2008; 52: 302-306.

35. O'Flaherty D, Husain MM, Moore $\mathrm{M}$, et al. Circulatory responses during electroconvulsive therapy: comparative effects of placebo, esmolol and nitroglycerin. Anaesthesia 1992; 47: 563-567.

36. Villalonga A, Planella T, Castillo $\mathrm{J}$, et al. Nitroglycerin spray in the prevention induced by electroconvulsive therapy. Rev Esp Anestesiol Reanim 1989; 36: 264-266.

37. Mokriski BK, Nagle SE, Papuchis $\mathrm{GC}$, et al. Electroconvulsive therapy-induced cardiac arrhythmias during anesthesia with methohexital, thiamylal, or thiopental sodium. J Clin Anesth 1992; 4: 208-212.

38. Rasmussen P, Andersson JE, Koch $\mathrm{P}$, et al. Glycopyrrolate prevents extreme bradicardia and cerebral deoxygenation during electroconvulsive therapy. J ECT 2007; 23: 147-152.

39. Avramov MN, Husain MM, White PF. The comparative effects of methohexital, propofol, and etomidate for electroconvulsive therapy. Anesth Analg 1995; 81:596-602.

40. Geretsegger C, Nickel M, Ju- 
dendorfer B, et al. Propofol and methohexital as anesthetic agents for electroconvulsive therapy: a randomized double blind comparison of electroconvulsive therapy seizure quality, therapeutic efficacy, and cognitive performance. J ECT 2007; 23: 239-243.

41. Graveland P, Wierdsma A, Van den Broek W, et al. A retrospective comparison of the effects of propofol and etomidate on stimulus variables and efficacy of electroconvulsive therapy in depressed inpatients. Prog Neuropsychopharmacol Biol Psychiatry 2013; 45: 230-235.

42. Fredman B, D'Etienne J, Smith I, et al. Anesthesia for electroconvulsive therapy: effects of propofol and methohexital on seizure activity and recovery. Anesth Analg 1994; 79: 75-79.

43. Vaidya PV, Anderson EL, Bobb
A, et al. A within-subject comparison of propofol and methohexital anesthesia for electroconvulsive therapy. J ECT 2012; 28: 14-19.

44. Saito S, Kadoi Y, Nara T, et al. The comparative effects of propofol versus thiopental on middle cerebral artery blood flow velocity during electroconvulsive therapy. Anesth Analg 2000; 91: 1531-1536.

45. Saffer S, Berk M. Anesthetic induction for ECT with etomidate is associated with longer seizure duration than thiopentone. J ECT 1998; 14: 89-93.

46. Begec Z, Erdogan G, Toprak HI, et al. Sevoflurane alone and propofol with or without remifentanil for electroconvulsive therapy-a randomised, crossover study. Anaesth Intensive Care 2013; 41: 202-206.

47. Mirzakhani H, Welch CA, Eikermann M, et al. Neuromuscular blocking agents for electroconvul- sive therapy: a systematic review. Acta Anaesthesiol Scand 2012; 56: 3-16.

48. Kramer BA. Use of ECT in California, revisited: 1984-1994. J ECT 1999; 15: 245-251.

49. Angst J, Angst K, Baruffol I, et al. ECT-induced and drug-induced hypomania. Convuls Ther 1992; 8 (3):179-185.

50. Rose D, Fleischmann P, Wykes T, et al. Patients' perspectives on electroconvulsive therapy: systematic review. BMJ 2003; 326: 1363.

51. Lisanby SH, Maddox JH, Prudic J, et al. The effects of electroconvulsive therapy on memory of autobiographical and public events. Arch Gen Psychiatry 2000; 57: 581.

52. González A, Cortinez L, De la Cuadra J. Anestesia en terapia electroconvulsiva. Experiencia clínica. Rev Esp Anestesiol Reanim 2007; 54: 414-420.

\section{Correspondencia a: \\ Fernando Reyes Roldán \\ Alejandro González Arellano fernando.ireyesr@gmail.com gonzalea@med.puc.cl}

